\title{
AP081: The impact of implementation of ReSPECT (Recommended Summary Plan for Emergency Care and Treatment) on the making of treatment escalations in patients with do not attempt cardiopulmonary resuscitation decisions.
}

Luke Jeyes $^{1}$, Michelle Hall ${ }^{1}$, Paul Slater ${ }^{1}$, Kathrine Broderick ${ }^{1}$, Keith Couper ${ }^{1,2}$, Gavin Perkins ${ }^{1,2}$ 1 University Hospitals Birmingham NHS Trust, Birmingham, UK.

2University of Warwick, Coventry, UK.

\section{Purpose of Study}

The Recommended Summary Plans for Emergency Care and Treatment (ReSPECT) programme aims to integrate resuscitation decision-making in a broader review of treatments that could be offered in an emergency. Historically when making resuscitation decisions, these have been made in isolation to other treatment considerations, leading to problems (Pitcher et al, 2017). A national working group led by the Resuscitation Council (UK), developed ReSPECT. The purpose of the study was to explore the impact of implementation of ReSPECT on the emergency care treatment plans in a hospital trust in the UK.

\section{Materials and Methods}

The study was designed as a before and after cohort study and used data from an existing quality improvement audit at 3 hospitals within University Hospitals Birmingham. A purposive sample comprising 2 years of audit data prior to and 2 years after the implementation of ReSPECT was examined. The frequency with which advanced emergency care decisions were made and whether the decisions related solely to resuscitation or consider overall emergency treatment plans were compared. Differences in proportions between periods were compared using Chi-squared test.

\section{Results}

In the 2 years prior to implementation of ReSPECT (2015-16), $15.2 \%$ ( $n=356 / 2349)$ of notes reviewed had an emergency care treatment decision. Post implementation of ReSPECT (201718 ), this figure had significantly increased to $18.8 \%$ $(n=437 / 2329, p<0.01)$.
Prior to implementation of ReSPECT, only $43 \%$ ( $n=152 / 356)$ recorded a do not attempt cardiopulmonary resuscitation decision, with no other documentation of a decision regarding other treatment escalation plans or limitations. After implementation of ReSPECT, a higher proportion, $86 \%$ $(n=378 / 437), p<0.0001)$ of records provided information on overall treatment escalation plans or limitations in addition to whether resuscitation should be attempted.

\section{Conclusion}

In a large hospital trust in the UK, implementation of ReSPECT increased both the numbers of advanced emergency treatment plans made, as well as the proportion of patients where a resuscitation decision was presented as part of a broader decisions about emergency treatment escalations and limitations.

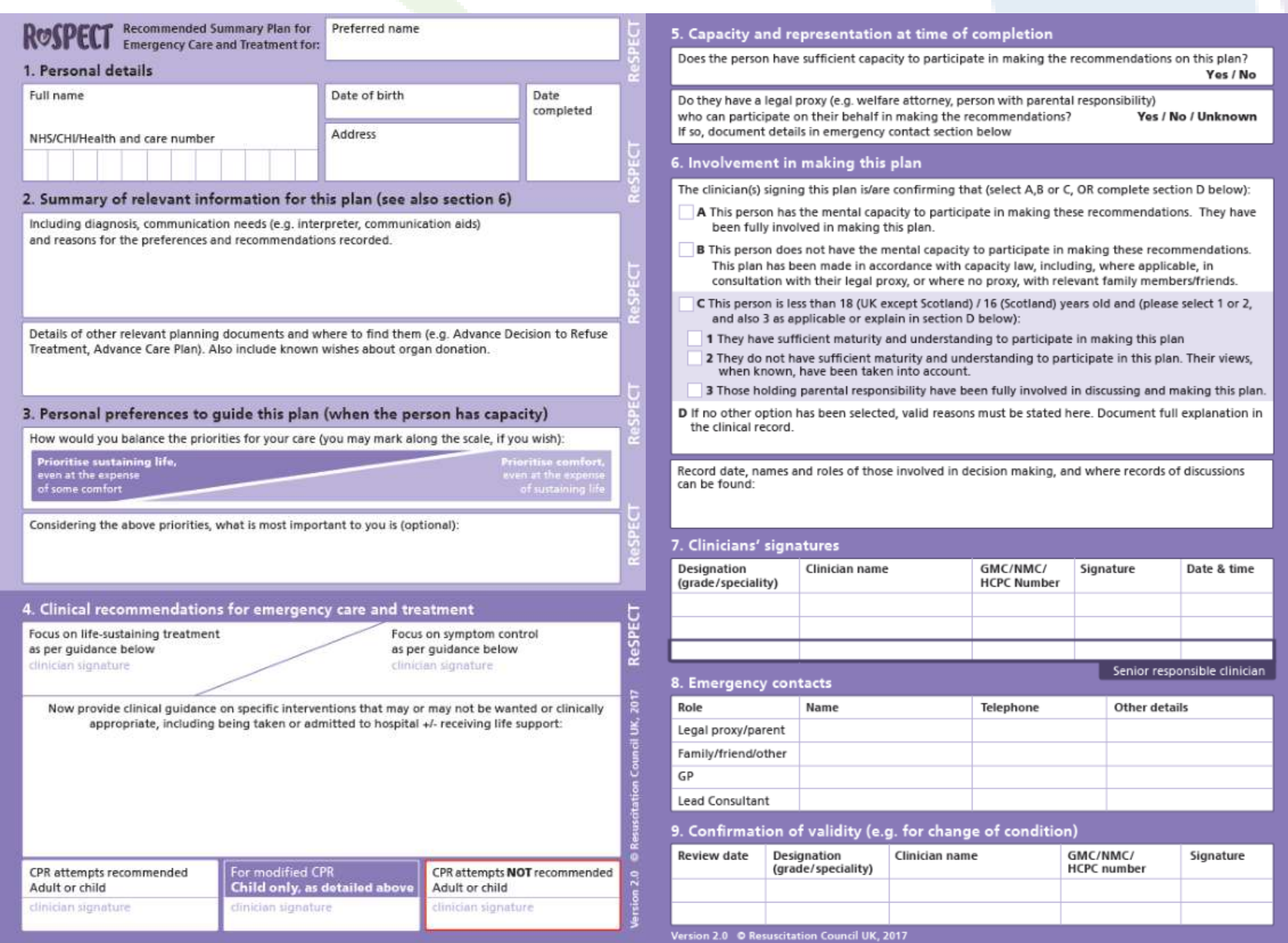

\section{References}

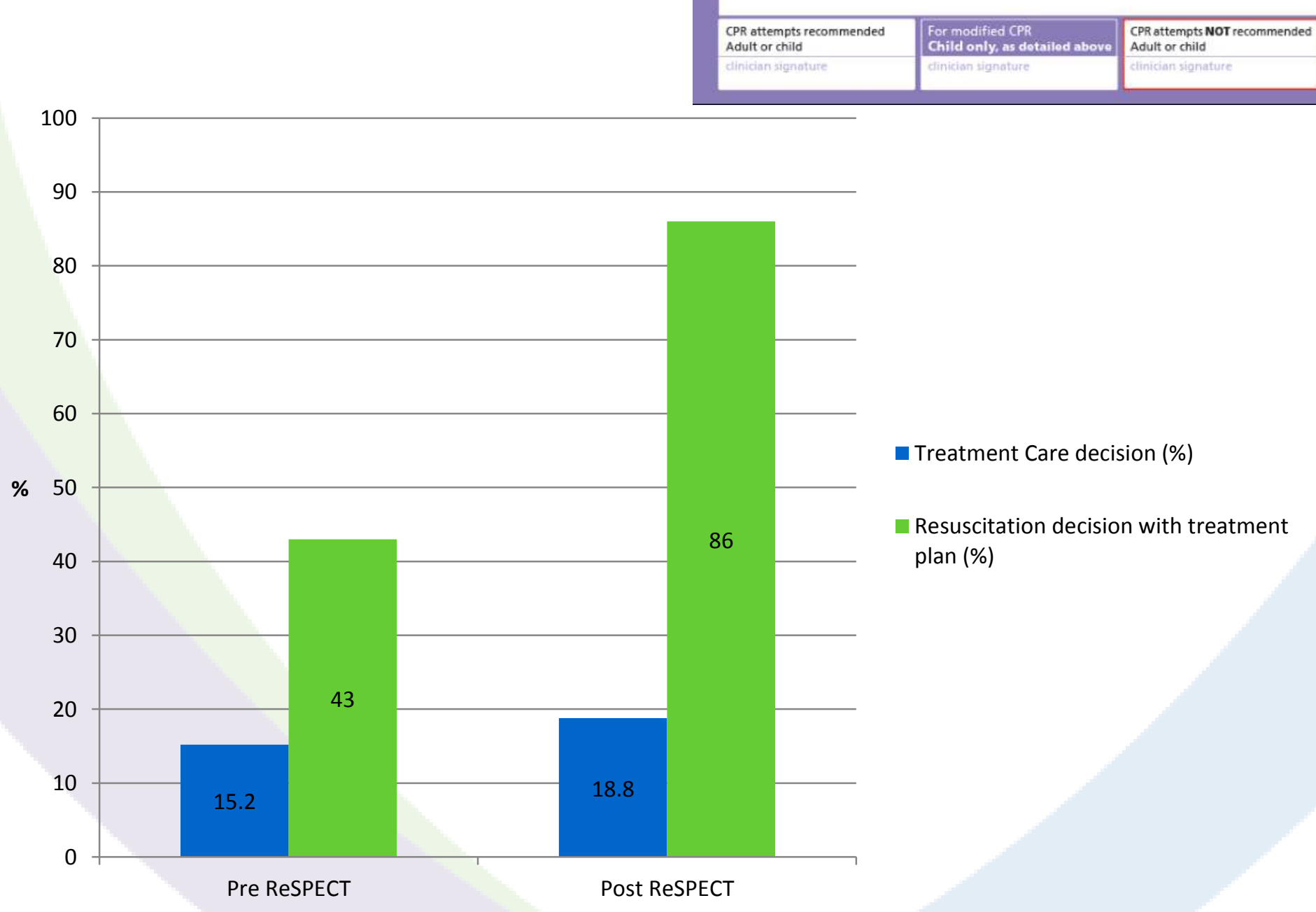

Pitcher, D., Fritz, Z., Wang, M., Spiller, J. (2017). Emergency Care and Resuscitation Plans. BMJ. Vol. 356. No. 8096. 